\title{
Nonlinearity Management: From Fiber Oscillators to Amplifiers
}

\author{
F. Ö. Ilday ${ }^{1,2}$, Ç. Şenel ${ }^{3}$, R. Hamid ${ }^{3}$, T. Teamir ${ }^{2}$, I. Pavlov ${ }^{2}$, U. Teğin, E. Ergeçen, P. Elahi, R. Iegorov ${ }^{2}$ \\ ${ }^{1}$ Department of Electrical and Electronics Engineering, Bilkent University, 06800, Ankara, Turkey \\ ${ }^{2}$ Department of Physics, Bilkent University, 06800, Ankara, Turkey \\ ${ }^{3}$ TÜBITTAK National Metrology Institute (UME), 41470, Kocaeli, Turkey
}

\begin{abstract}
While the standard approach to performance scaling in fiber lasers seeks to reduce nonlinear effects through chirping or mode scaling, I will review recent progress in a complementary approach, whereby the governing dynamics are meticulously exploited towards achieving superior performance.
\end{abstract}

\section{INTRODUCTION}

There has been tremendous progress in the development of fiber lasers within the last decade. These lasers have diverse applications including optical frequency metrology [1], various industrial applications [2], microscopy [3], as well as fundamental interest into their rich governing dynamics [4,5]. The progress in understanding the dynamics and technical development both have to address their extremely strong nonlinearities.

There are two basic approaches to managing nonlinear effects in lasers. The most commonly used and most successful way is to seek to minimize the nonlinear effects in the first place, either by stretching the pulses in the time domain using an implementation of chirped pulse amplification (CPA) [6], divided pulse amplification [7] or by increasing mode area of the fibers $[2,8,9]$. Despite being very effective, this approach is ultimately limited, as it does not actually solve the nonlinearity problem. Practical drawbacks include, e.g., the fact that the largestdiameter fibers are all rod-type fibers, which are rigid, which is a substantial step back in terms of integrability.

The second approach involves management of nonlinear effects, examples of which include use of negative (self-defocussing) nonlinearities [10,11], selfsimilar pulse propagation [12,13], dissipative soliton propagation $[4,14]$ nonlinear CPA [15].

Given that the approaches based on minimizing and managing nonlinearity can usually be combined very effectively, these two approaches should be regarded as complementary and not as alternatives. However, Nonlinearity Management [10] approach has been studied and exploited to a much lesser extend, despite holding great promise for further progress. In this contribution, we will provide a summary of recent progress and motivation for the Nonlinearity Management approach.

\section{NEW SCHEMES OF NONLINEARITY MANAGEMENT}

We and several other groups have been developing various ways of implementing Nonlinearity Management. Some of these are discussed below.

\section{A. Raman Fiber Oscillators}

Use of Raman gain to generate laser action dates back to 1977 [16] and first generation of solitons to 1987 [17]. However, these lasers generated extremely low-energy pulses and typically $\mathrm{km}$-long cavities. Similaritons have been used to generate energetic pulses, but remained in the picosecond duration [18]. The potential impact is substantial, if multi-nJ and femtosecond pulses can be generated, because, despite major improvements in fiber laser performance in the last decade, there has been little improvement in terms of wavelength coverage, which is increasingly important especially for imaging [3], but also for spectroscopy.

Recently, we have demonstrated what we believe to be the first true Raman dissipative-soliton in a fiber resonator [19]. This laser differed fundamentally from the case of [20], in that only the Raman pulses (the Stokes component) was resonant and propagating through the cavity. This work was followed upon by [21], achieving similar results. Based on the promising results that have already been demonstrated, it is clear that further progress on dissipative-soliton Raman oscillators have great potential in extending high-energy, femtosecond-pulsed mode-locked laser performance to wavelengths beyond the traditional gain media of Er, Yb and Tm.

We will present a theoretical model on how the balance of the nonlinear effects occurs in the cavity and how this contributes to the formation stable, synchronized pulses.

\section{B. Spectral Sculpturing}

The consequences of nonlinear effects are most vividly visible in the spectral domain, through the creation, erasure or conversion of spectral portions. This suggests that managing nonlinear effects may be achieved by imposing amplitude or phase modulations on the spectrum. This is already commonly used in the form of fixed spectral filters to influence mode-locking and pulse propagation $[14,22]$, but a more versatile possibility is to use freely configurable filters. Despite technical difficulties, this is possible with a spatial light modulator, which we have shown for the first time inside of a modelocked laser cavity. Using this approach, we are able to directly influence the mode-locking, help it initiate, clean up undesirable features such as $\mathrm{CW}$ peaks and broaden the mode-locked spectrum. Future work in this direction can include combination with advanced algorithms for mode-locking control as demonstrated in [23]. 


\section{Effective Negative Nonlinearity}

While the most direct approach to managing nonlinearity in mode-locked oscillators is to use selfdefocusing $\left(n_{2}<0\right)$, as proposed in [10], it is not easy to achieve this in practice with cascaded quadratic processes, although a recent demonstration has been made, constituting a major step forward [11].

There is alternative approach, which allows judicious use of normal dispersion, spectral filtering (which can be from gain narrowing) and positive nonlinearity that imparts an effectively negative nonlinearity [24]. We will present a mode-locked oscillator, which incorporates using this approach for the first time to our knowledge.

\section{Amplifiers Designed Like Oscillators}

While the techniques of Nonlinearity Management that we have discussed above pertain to mode-locked laser oscillators, it is highly useful to apply concepts from mode-locking to amplifiers as well, which has not been extensively explored to date.

What makes mode-locking both difficult to achieve, characterize and theoretically model, as well as dynamically rich is that evolution is subject to feedback by virtue of the laser cavity, which imposes periodic boundary conditions on all solutions. In other words, the pulse shape must exactly return to its original state at the end of each roundtrip, for the laser to be operating stably. A well-designed oscillator has its dynamics such that there is an island of stability (a basin of attraction) around each solution, so that any perturbations decay. This property can be exploited to design highly robust and versatile pulse evolution schemes for amplifier chains, even though the output of the amplifier never needs to evolve back to its input signal. However, we can design the amplifier with hypothetical sections, such as passive propagation, filtering, that, if placed after the amplifier, would allow fulfilling the periodicity condition. Since these sections will not be physically constructed, they do not need to have realistic parameters either, but they serve as mathematical tools enabling discovery highly robust solutions to difficult problems, such as maintaining a broad spectrum after amplification: An fiber oscillator can have $20 \mathrm{~dB}$ gain per roundtrip, but can be configured to support 50-fs pulses [25], which is non-trivial in an amplifier stage. We will demonstrate the use of this approach in designing a multi-stage amplifier that balances gain narrowing with self-phase modulation, very similar to dynamics inside of a mode-locked oscillator.

\section{CONCLUSIONS}

Nonlinearity Management has emerged as a powerful approach to designing high-performance ultrafast fiber oscillators and amplifiers. It is complementary to and can be combined with the commonly used techniques for reduction of nonlinearities, such as chirping, use of largecore fibers, divided pulse amplification. This approach exploits the dynamics of pulses, principles of which are well known, but it is necessary to combine the cavity elements in clever ways to achieved the desired effect. Each approach that falls under Nonlinearity Management is born from improved understanding of mode-locking physics. This point beautifully highlights the highly synergistic and intimate link between fundamental studies of mode-locking and ultrafast pulse propagation, motivated primarily by the underlying physics and practical, technological progress in ultrafast fiber lasers and amplifiers. This talk will review the aforementioned set of activities and approaches developed in our group as well as other groups worldwide.

\section{ACKNOWLEDGMENT}

This work was supported by the European Research Council (ERC) Consolidator Grant ERC-617521-NLL, and TÜBITAK under projects 112T980, 112T944, and $113 \mathrm{~F} 319$.

\section{REFERENCES}

[1] M. E. Fermann and I. Hartl, Nature Photon. 7, 868 (2013).

[2] C. Jauregui, J. Limpert, and A. Tünnermann, Nature Photon. 7, 861 (2013).

[3] C. Xu and F. W. Wise, Nature Photon. 7, 875 (2013).

[4] P. Grelu and N. Akhmediev, Nature Photon. 6, 84 (2012).

[5] E. G. Turitsyna, S. V. Smirnov, S. Sugavanam, N. Tarasov, X.

Shu, S. A. Babin, E. V. Podivilov, D. V. Churkin, G. Falkovich, and

S. K. Turitsyn, Nature Photon. 7, 783 (2013).

[6] D. Strickland and G. Mourou, Opt. Comm. 56, 219 (1985).

[7] S. Zhou, F. W. Wise, and D. G. Ouzounov, Opt. Lett. 32, 871 (2007).

[8] M. E. Fermann, Opt. Lett. 23, 52 (1998).

[9] G. Mourou, B. Brocklesby, T. Tajima, and J. Limpert, Nature Photon. (2013).

[10] F. Ö. Ilday and F. W. Wise, J. Opt. Soc. Am. B 19, 470 (2002).

[11] C. R. Phillips, A. S. Mayer, A. Klenner, and U. Keller, Optica, Optica 2, 667 (2015).

[12] M. Fermann, V. Kruglov, B. Thomsen, J. Dudley, and J. Harvey, Phys. Rev. Lett. 84, 6010 (2000).

[13] F. Ö. Ilday, J. R. Buckley, W. G. Clark, and F. W. Wise, Phys. Rev. Lett. 92, 213902 (2004).

[14] W. H. Renninger, A. Chong, and F. W. Wise, Opt. Lett. 33, 3025 (2008).

[15] S. Zhou, L. Kuznetsova, A. Chong, and F. Wise, Opt. Express 13, 4869 (2005).

[16] R. H. Stolen, C. Lin, and R. K. Jain, Appl. Phys. Lett. 30, 340 (1977).

[17] J. D. Kafka and T. Baer, Opt. Lett. 12, 181 (1987).

[18] C. Aguergaray, D. Méchin, V. Kruglov, and J. D. Harvey, Opt. Express 18, 8680 (2010).

[19] U. Tegin, P. Elahi, S. Yilmaz, and F. Ö. Ilday, in Europhoton (Neuchatel, 2014).

[20] S. A. Babin, E. V. Podivilov, D. S. Kharenko, A. E. Bednyakova, M. P. Fedoruk, V. L. Kalashnikov, and A. Apolonski, Nat. Comm. 5, 1 (1AD).

[21] D. Churin, J. Olson, R. A. Norwood, N. Peyghambarian, and K. Kieu, Opt. Lett. 40, 2529 (2015).

[22] B. Öktem, C. Ülgüdür, and F. Ö. Ilday, Nature Photon. 4, 307 (2010).

[23] U. Andral, R. S. Fodil, F. Amrani, F. Billard, E. Hertz, and P. Grelu, Optica, Optica 2, 275 (2015).

[24] D. N. Schimpf, E. Seise, J. Limpert, and A. Tuennermann, Opt. Express 17, 4997 (2009).

[25] F. O. Ilday, J. R. Buckley, H. Lim, F. W. Wise, and W. G. Clark, Opt. Lett. 28, 1365 (2003). 